\title{
Feature related multi-view nonnegative matrix factorization for identifying conserved functional modules in multiple biological networks
}

\author{
Peizhuo Wang, Lin Gao* (D), Yuxuan Hu and Feng Li
}

\begin{abstract}
Background: Comprehensive analyzing multi-omics biological data in different conditions is important for understanding biological mechanism in system level. Multiple or multi-layer network model gives us a new insight into simultaneously analyzing these data, for instance, to identify conserved functional modules in multiple biological networks. However, because of the larger scale and more complicated structure of multiple networks than single network, how to accurate and efficient detect conserved functional biological modules remains a significant challenge.

Results: Here, we propose an efficient method, named ConMod, to discover conserved functional modules in multiple biological networks. We introduce two features to characterize multiple networks, thus all networks are compressed into two feature matrices. The module detection is only performed in the feature matrices by using multi-view non-negative matrix factorization (NMF), which is independent of the number of input networks. Experimental results on both synthetic and real biological networks demonstrate that our method is promising in identifying conserved modules in multiple networks since it improves the accuracy and efficiency comparing with state-of-the-art methods. Furthermore, applying ConMod to co-expression networks of different cancers, we find cancer shared gene modules, the majority of which have significantly functional implications, such as ribosome biogenesis and immune response. In addition, analyzing on brain tissue-specific protein interaction networks, we detect conserved modules related to nervous system development, mRNA processing, etc.
\end{abstract}

Conclusions: ConMod facilitates finding conserved modules in any number of networks with a low time and space complexity, thereby serve as a valuable tool for inference shared traits and biological functions of multiple biological system.

Keywords: Features, Multiple biological networks, Conserved modules, Matrix factorization

\section{Background}

Recent high-throughput experimental techniques brought a large number of multi-omics data (e.g., DNA sequence data, mRNA, miRNA, methylation, copy number variation, etc.) in different conditions (e.g., tissue types and disease states). Comprehensive analysis of these multiple biological data is non-trivial for more profound understanding of the whole biological system

\footnotetext{
* Correspondence: Igao@mail.xidian.edu.cn

School of Computer Science and Technology, Xidian University, Xi'an 710071,
} China

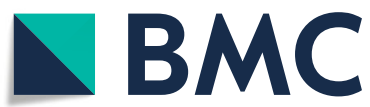

(0) The Author(s). 2018 Open Access This article is distributed under the terms of the Creative Commons Attribution 4.0 International License (http://creativecommons.org/licenses/by/4.0/), which permits unrestricted use, distribution, and

reproduction in any medium, provided you give appropriate credit to the original author(s) and the source, provide a link to the Creative Commons license, and indicate if changes were made. The Creative Commons Public Domain Dedication waiver (http://creativecommons.org/publicdomain/zero/1.0/) applies to the data made available in this article, unless otherwise stated.
[1]. As a promising tool for integrative analyzing large-scale biological data, network-based approach is successful in discovering biological meaning patterns. However, most of the network-based works only concern single biological data that is insufficient to simultaneously analyze multi-omics or multiple conditions data and hinder us from capturing comprehensive information on total system. In order to settle this issue, more complex models, namely multiple networks or multi-layer network models [2, 3], have been introduced. The multiple networks, which can be created by 
incorporating multiple types of connection and constituting the environment to describe systems interconnected through different categories of connections, bring us a new insight into biological mechanism and medicine research in a comprehensive level $[4,5]$.

One significant task in multiple biological networks is to detect conserved functional modules, for the reason that the biological networks across different type of tissues, cancers or disease states have many shared patterns or underlying common cellular functional organizations, which can be represented as module structures. For example, cancers of disparate organs have many shared features [6], including rapid cell proliferation, the ability to migrate and avoiding immune destruction, etc. [7]. Understanding these common traits by identifying the underlying conserved function modules are key to gaining insight into cancer physiology and ultimately to prevent cancer. Moreover, as another example, identifying common features in biological networks across distant species can reveal evolvement relations and fundamental principles $[8,9]$.

Despite the great importance of extracting conserved modules in multiple biological networks, it is highly difficult to develop an effective and efficient algorithm because of two reasons. First, it is hard to characterize features of conserved modules due to the more complicated structure of multiple networks. Second, multiple networks pose a great challenge for designing efficient algorithms, since multiple networks have larger scale than single network and how to reduce time and space complexity is need to address. To handle these issues, a simple strategy is to summarize a collection of heterogeneous data into a single integrated network and use graph-based clustering on it. However, this strategy can bring about the substantial information loss. Recent years, researches developed methods on module discovery in multiple networks, such as a heuristic algorithm to mine frequent coherent dense subgraphs on unweighted networks [10], tensor based optimization algorithm [11], generalized singular decomposition based method [12], and modularity based optimization algorithm [9]. However, these methods are either limited to cluster on unweighted networks [10] or take a lot of time and memory for running $[9,11,12]$. Almost at the same time, the multi-view clustering approaches from machine learning field were also put forward to cluster for integrated data [13-16]. In these approaches, each data object is comprised of different representations (views) that provide compatible and complementary information for better clustering. However, most of these multi-view clustering methods assume that all views consist of the same set of data objects, which is not suitable to some circumstance. Moreover, these methods always separately analyze the structure of each network and concatenate the results, which greatly increase the dimensionality of the space.

In this paper, we develop an approach, called ConMod, to discover Conserved functional Modules in multiple biological networks. Instead of mining each biological network individually, ConMod describes the networks as two feature matrices and performs a multi-view clustering approach based on non-negative matrix factorization (NMF) in these two matrices only. Our main contributions of the proposed approach are summarized as follows:

- We introduce two features to measure the strength and distribution of each edges in multiple networks. Thus, all of the multiple networks are compressed into two feature matrices, which is the basis of detecting conserved module with a low time and space complexity.

- We adopt a multi-view symmetric NMF model based on our proposed feature matrices, which help us find consensus factors with effectiveness and efficiency.

- Our method can discover conserved modules without denoting the number of networks that a module appears. If the overall signal in the consensus factors is detected, a conserved module will be found. The results show that our method can accurate find modules that appear in more than half of all networks.

To show substantial improvements over the state-of-the-art methods, we demonstrate ConMod's accuracy and efficiency to discover conserved modules from multiple networks in two types of synthetic datasets. Moreover, to verify the biological meaning of conserved modules, we apply ConMod in two distinct biological multiple networks: (1) 33 cancer type-specific gene co-expression networks and (2) 15 brain-specific protein interaction networks. Both two tasks demonstrate the potential to effectively identify conserved modules with significantly functional implications, such as DNA replication, ribosomal protein biosynthesis and immune response in 33 cancers' co-expression networks and nervous system development in 15 brain PPI networks, respectively. ConMod can be used to simultaneously analyze any number of networks and straightforwardly applied to other types of networks in addition to biology.

\section{Methods \\ Overview}

The multiple networks, or multi-layer network, with $M$ layers can be represented by the set $\mathcal{G}=\left\{G^{(1)}, G^{(2)}, \ldots\right.$, $\left.G^{(M)}\right\}$, whose element $G^{(t)}=\left(V^{(t)}, E^{(t)}, W^{(t)}\right)(t=1,2, \ldots, M)$ is an undirected network under consideration with vertex set $V^{(t)}$ and edge set $E^{(t)}$, where $N_{t}=\left|V^{(t)}\right|$ denotes 
the number of nodes in the network layer $t . G^{(t)}$ is represented by an $N_{t} \times N_{t}$ adjacency matrix $\mathbf{W}^{(t)}$, where each element $w_{i j}^{(t)}$ is the weight of the edge between nodes $i$ and $j$ in the network layer $t . N=\left|\cup_{t} V^{(t)}\right|$ is the total number of nodes in multiple networks.

The goal of our method ConMod is to identify the conserved functional modules, which exist in as many of the biological networks as possible. Figure 1 shows the flowchart of our method for detecting conserved functional modules. The basic framework of ConMod involves three steps. First, we transform multiple networks into two feature matrices, the connection strength matrix and the participation coefficient matrix, which respectively describes the overall edge weight and the degree of participation of each edge in multiple networks. Second, we jointly factorize the two feature matrices into consensus factors by using multi-view NMF. Finally, we adopt a soft node selection procedure from the consensus factors to assign the module members and then we refine the candidate modules for obtaining more accurate results. We implemented ConMod in MATLAB R2015a as a user-friendly package (https://github.com/WPZgithub/ConMod).
Transforming multiple networks into two feature matrices For multiple networks, conserved modules not only have densely topological structure in each network, but also broadly distribute in most networks. Based on this point, we propose two features to describe a conserved functional module. The first, connection strength, is used for characterizing whether a pair of nodes connect closely in multiple networks. The second, participation coefficient, is used for describing whether an edge is uniformly distributed across all networks. In this way, the conserved modules detection is equivalent to find node sets that consist of the edges with high connection strength and participation coefficient.

The connection strength of an edge between nodes $i$ and $j$, denoted as $x_{i j}^{(s)}$, is defined as the average weight over all networks:

$$
x_{i j}^{(s)}=\frac{\sum_{t=1}^{M} w_{i j}^{(t)}}{M} .
$$

In addition, we define the participation coefficient of an edge, denoted as $x_{i j}^{(p)}$, as following:

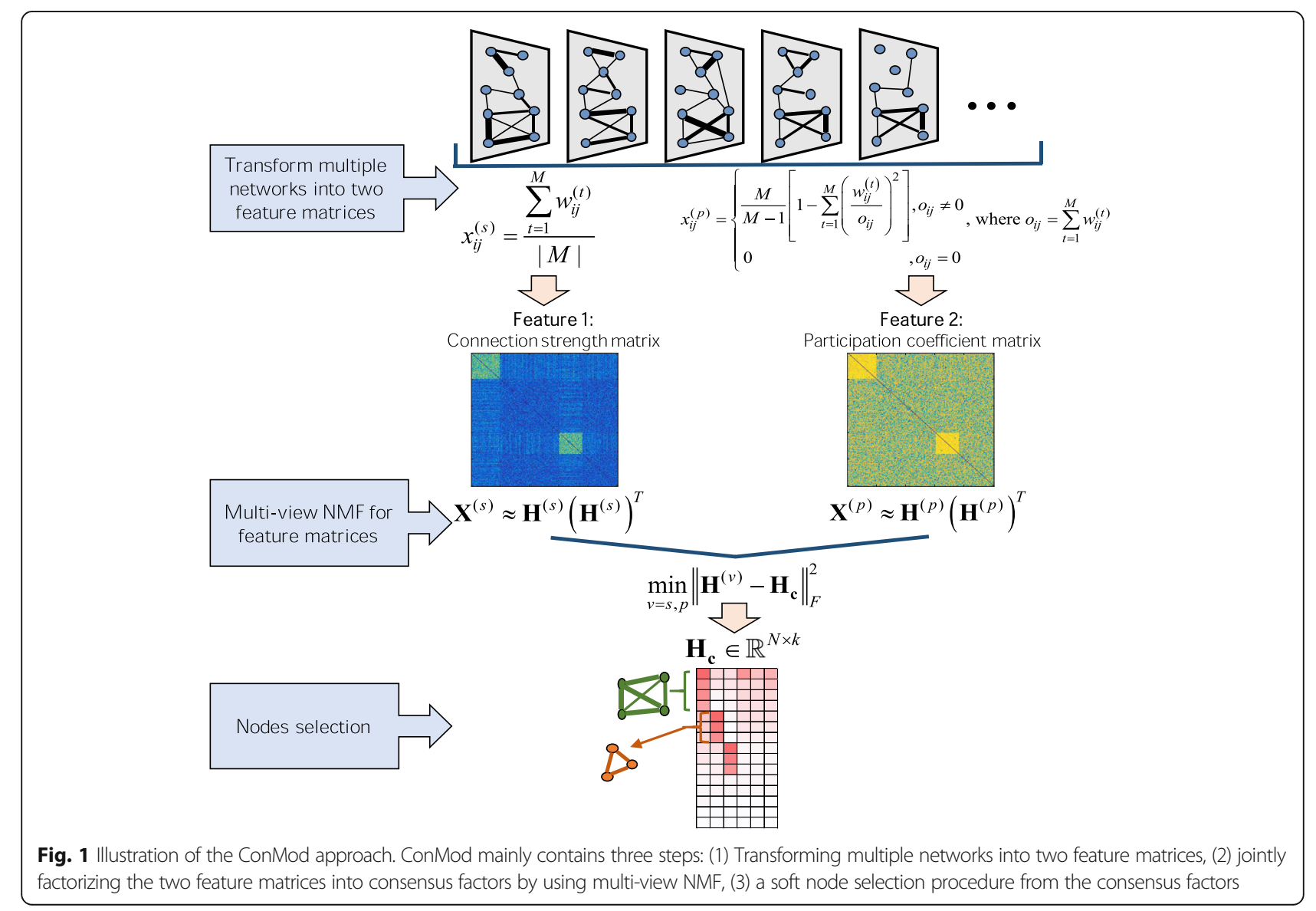




$$
x_{i j}^{(p)}=\left\{\begin{array}{c}
\frac{M}{M-1}\left[1-\sum_{t=1}^{M}\left(\frac{w_{i j}^{(t)}}{o_{i j}}\right)^{2}\right], o_{i j} \neq 0 \\
0, o_{i j}=0
\end{array}\right.
$$

where $o_{i j}=\sum_{t} w_{i j}^{(t)}$. The definition of the participation coefficient is first introduced by Guimera and Amaral $[17,18]$ to quantify the participation of a node to the different communities of a network. In our paper, we change it to measure edges and adapt it to multiple networks. Here, the participation coefficient measures whether an edge uniformly distributed among the $M$ networks. The larger the value of the coefficient $x_{i j}^{(p)}$ is, the more uniformly distributed the edge will be in the multiple networks.

Both values of the connection strength and the participation coefficient are in $[0,1]$. These two features can be used for both weighted and unweighted networks. However, for weighted networks, direct calculation of the participation coefficient for each weighted edge may not be appropriate, since the huge quantity of weakly connected edges may have very high value of participation coefficient. For example, if $w_{i j}^{(t)}=0.01$ for all $t=1,2, \cdots$, $M$, the participation coefficient $x_{i j}^{(p)}=1$, but the edges between nodes $i$ and $j$ are most likely to be neglected for module discovery due to the very low edge weight. Even though the connection strength $x_{i j}^{(s)}$ is small enough, the high value of participation coefficient will increase noise for conserved module detection. To handle this issue, we take the logistic transform of the input data and neglect the edges with low transformed values. Specifically, for weighted networks, the original adjacency matrix of each network is first transformed using a logistic function $L\left(w_{i j}\right)=1 /\left(1+\exp \left(c w_{i j}+d\right)\right)$, such that for $w_{i j} \in$ $[0,0.3], L\left(w_{i j}\right) \approx 0$, and for $w_{i j} \in[0.6,1], L\left(w_{i j}\right) \approx 1$. This implies that $L(0)$ needs to be close to 0 . So we first normalize the adjacent matrix such that each element of the matrix is in $[0,1]$ and then we set $L(0)=0.0001$, from which we obtain $d=\log (9999)$ and $c=-2 \log (9999)$.

\section{Computing consensus factors using multi-view symmetric NMF based on feature matrices}

From now on, the relationships among $N$ nodes are represented by 2 -view representations, $\mathbf{X}^{(s)}$ and $\mathbf{X}^{(p)}$. Now we cluster across the two views simultaneously to find a common latent structure. Among the multi-view clustering algorithms, NMF based methods $[14,19,20]$ have demonstrated strong vitality and efficiency. Based on the two feature matrices we use a multi-view NMF model [14] to find a common coefficient (or basis) matrix. Here, the original multi-view NMF model is adjusted for handling our symmetric feature matrices. Thus, we have the following objective function of the multi-view symmetric NMF:

$$
\begin{aligned}
& \min _{\mathbf{H}^{(v)}, \mathbf{H}_{\mathrm{c}}} \mathcal{F}=\left(\sum_{v=s, p}\left\|\mathbf{X}^{(v)}-\mathbf{H}^{(v)}\left(\mathbf{H}^{(v)}\right)^{T}\right\|_{F}^{2}+\sum_{v=s, p} \lambda_{v}\left\|\mathbf{H}^{(v)}-\mathbf{H}_{\mathbf{c}}\right\|_{F}^{2}\right) \\
& \text { s.t. } \mathbf{H}^{(v)} \geq 0, \mathbf{H}_{\mathbf{c}} \geq 0
\end{aligned}
$$

where $\|\cdot\|_{F}$ denotes Frobenius norm and $\lambda_{\nu}$ is the parameter to balance the relative weight of different views. The multi-view symmetric NMF factorize each view of symmetric data matrix $\mathbf{X}^{(\nu)}$ to a low-rank matrix representation $\mathbf{H}^{(v)}$, which are close to the consensus matrix $\mathbf{H}_{\mathbf{c}}$.

To solve this optimization problem, we use the multiplicative update rule to minimize the objection function. Specifically, given a desired rank $k$, the algorithm iterates the following two steps until convergence. First, we fix $\mathbf{H}_{\mathbf{c}}$ and minimize objective function over $\mathbf{H}^{(v)}$ for each view $v . \mathbf{H}^{(\nu)}$ is updated at each step by:

$$
\left(\mathbf{H}^{(v)}\right)_{i k} \leftarrow\left(\mathbf{H}^{(v)}\right)_{i k} \frac{\left(2 \mathbf{X}^{(v)} \mathbf{H}^{(v)}+\lambda_{\nu} \mathbf{H}_{\mathbf{c}}\right)_{i k}}{\left(2 \mathbf{H}^{(v)}\left(\mathbf{H}^{(v)}\right)^{T} \mathbf{H}^{(v)}+\lambda_{v} \mathbf{H}^{(v)}\right)_{i k}}, v=s, p .
$$

Second, fixing $\mathbf{H}^{(v)}$ for each $v$, we take the derivative of the objective $\mathcal{F}$ over $\mathbf{H}_{\mathbf{c}}$ and obtain an exact solution:

$$
\mathbf{H}_{\mathbf{c}}=\frac{\sum_{v=s, p} \lambda_{\nu} \mathbf{H}^{(v)}}{\sum_{v=s, p} \lambda_{v}} \geq 0 .
$$

Since the objective function is non-convex, one should perform many repetitions and choose the minimizer of the objective function as the final solution.

\section{Selecting nodes from the consensus factors}

Once the consensus matrix $\mathbf{H}_{\mathbf{c}}$ is obtained, the cluster label of data point $i$ could be computed as arg$\max _{k}\left(\mathbf{H}_{\mathbf{c}}\right)_{i, k}$. However, it will be meaningless to use this hard clustering process in most biological networks. In gene networks, for instance, some genes are multifunctional, such as the broadly expressed transcription factors and the crosstalk of gene pathways. Besides, some genes are inactive in any module in some specific conditions. Therefore, we adopt a soft node selection procedure to obtain modules with biological meaning. The nodes are selected if they have relatively large absolute values of the weighted factors $\mathbf{H}_{\mathbf{c}}$. Specifically, we calculated the $z$-score for each column of $\mathbf{H}_{\mathbf{c}}$ by. 


$$
z_{i j}=\frac{\left(\mathbf{H}_{\mathbf{c}}\right)_{i j}-\mu_{\left(\mathbf{H}_{\mathbf{c}}\right)_{j}}}{\sigma_{\left(\mathbf{H}_{\mathbf{c}} \cdot{ }_{j}\right.}},
$$

where $\quad \mu_{\left(\mathbf{H}_{\mathbf{c}}\right)_{j}, j}=\frac{1}{N} \sum\left(\mathbf{H}_{\mathbf{c}}\right)_{i j} \quad$ and $\quad \sigma_{\left(\mathbf{H}_{\mathbf{c}}\right)_{j},}^{2}=\frac{1}{N-1} \sum$ $\left(\left(\mathbf{H}_{\mathbf{c}}\right)_{i j}-\mu_{\left.\left(\mathbf{H}_{\mathbf{c}}\right)_{j}\right)^{2}}{ }^{2}\right.$. We assign node $i$ as a member of a module, if $z_{i j}>\theta$. The threshold $\theta$ is typically in $[2,5]$ for most cases such that the selected nodes have significant signals in the consensus factors.

Finally, two modules with $\frac{\left|C_{x} \cap C_{y}\right|}{\min \left\{\left|C_{x}\right|,\left|C_{y}\right|\right\}}>0.5$ are merged and the modules whose sizes are smaller than five are removed, where $C_{x}$ is the members set of module $x$.

\section{Complexity analysis}

We first discuss the time complexity of our method. If the input networks are in the form of full matrix, the time complexity of computing two feature matrices is constant. While if the input networks are in the form of sparse matrix, its time complexity is $O(M e)$, where $e$ is the average number of edges of each network. Moreover, the time cost of the multi-view NMF procedure is $O\left(l k N^{2}\right)$, where $l$ is the number of iterations. The time complexity of selecting nodes from consensus factors is $O(k N)$. Therefore, the overall time cost is $O(M e)+$ $O\left(l k N^{2}\right)+O(k N)$. Since $N-1 \leq e \leq N(N-1) / 2$, then the total time complexity of ConMod is $O\left((l k+M / 2) N^{2}\right)$ in the worst case and $O\left(l k N^{2}\right)$ in the best case, demonstrating the efficiency of our method.

Then we discuss the space complexity. Multiple networks $\mathcal{G}=\left\{G^{(1)}, G^{(2)}, \ldots, G^{(M)}\right\}$ requires space $O\left(N^{2} M\right)$. However, our method compress the multiple networks into two feature matrices, and use multi-view NMF for conserved module detection, whose space complexity is $O\left(2 N^{2}\right)$ and $O(2 N k)$, respectively. Thus, the overall space complexity of ConMod is $O\left(2 N^{2}\right)$, which has nothing to do with the number of networks, demonstrating the efficiency of our approach on space complexity.

\section{Module validation}

We use a permutation test to assess the significance of functional modules across multiple networks. This allows identifying the specific conditions where each module is detected. Here, we use the cluster quality [12] as a measurement to calculated a $p$-value indicating the significance of one module in each network. The cluster quality is defined as:

$$
q_{t}=\frac{\text { the density within the module in } G^{(t)}}{\text { the density outside the module in } G^{(t)}} .
$$

The $p$-value is computed as the proportion of the random modules with the cluster quality larger than $q_{t}$. Raw $p$-values are corrected by using the method of
Benjamin-Hochberg [21] and the corrected $p$-values below 0.01 are regarded as significant existing of a module in a specific network.

\section{Results and discussion}

In this section, we first present simulation studies to demonstrate the performance of ConMod to detect conserved modules in synthetic multiple networks. We compare ConMod with four state-of-the-art methods, including NetsTensor [11], SC-ML [16], multi-view pairwise co-regularized spectral clustering (pairwiseCRSC) [15] and multi-view centroid-based co-regularized spectral clustering (centroidCRSC) [15]. NetsTensor introduced a tensor-based computational framework to identify recurrent heavy subgraphs in multiple biological networks. SC-ML modeled each graph layer as a subspace on a Grassmann manifold and then efficiently merge these subspaces find a unified clustering of the vertices. PairwiseCRSC and centroidCRSC employed a spectral clustering-based co-regularization framework for clustering across multiple views. Furthermore, to test whether ConMod is effective for finding conserved modules with meaningful biological functions, we apply ConMod to two sets of real biological networks, a set of 33 cancer type-specific gene co-expression networks and a set of human 15 brain tissue-specific protein interaction networks.

\section{Results on synthetic networks Simulation data}

To test the performance, we first evaluate our method using synthetic networks. We generate two sets of synthetic networks that contain different types of conserved modules: (1) conserved modules are common to a given set of networks and (2) conserved modules are present only in a subset of networks and they are the overlapping parts of specific modules across different networks.

We consider the first type of synthetic multiple networks with $M=30$ networks and $N=500$ nodes. We generate five modules with 80 nodes in each module and these modules are randomly assigned into $25,20,15,10$ and 5 networks, respectively. In this way, each network contains up to five modules. In each network, we connect nodes with a possibility of $\alpha(0<\alpha<1)$ inside each module and the nodes belonging to different modules are connected with a possibility of $\beta(0<\beta<\alpha)$. An example is shown in Fig. 2a. In order to introduce edge weights, we embed Gaussian noise on the networks (See more details in Additional file 1).

For the second type of synthetic dataset, we consider multiple networks with $M=15$ networks and $N=500$ nodes. In each network, a module consists of two parts, a common part, in which the nodes are common to a set of networks, and a specific part, in which nodes present only in its individual network. The common 


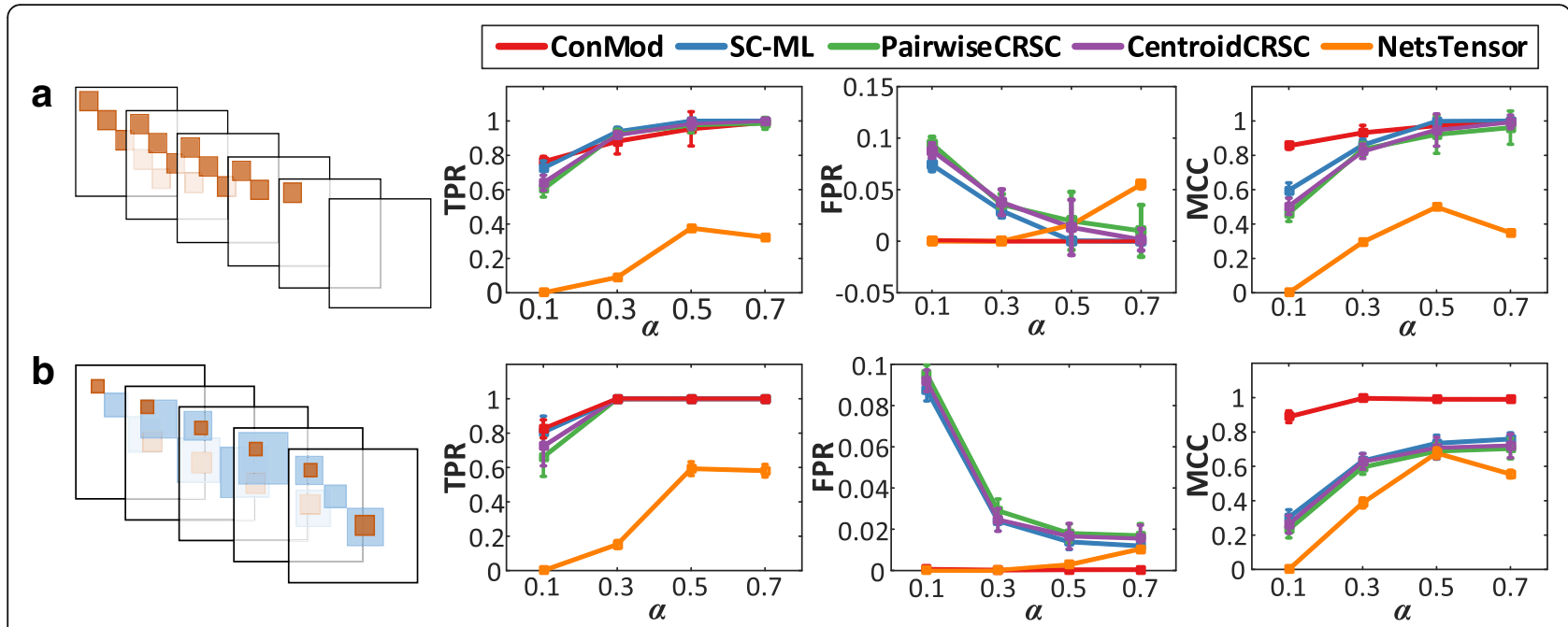

Fig. 2 Performance in terms of TPR, FPR and MCC with different $\alpha$. a The conserved modules are common to a given set of networks. $\mathbf{b}$ The conserved modules are the overlapping parts of specific modules across different networks

parts of every module are regarded as conserved modules in this case. We set two conserved modules of this type for this synthetic dataset. A conserved module has 50 nodes and another has 40 nodes. An example is shown in Fig. 2b. Other procedures for synthetic networks construction is the same as mentioned above (See more details in Additional file 1).

In this study, we experiment on synthetic networks with $\alpha=0.1,0.3,0.5$ and 0.7 and $\beta=0.05$. Lower value of $\alpha$ means modules are fuzzier and harder to detect.

\section{Evaluation measures}

We use true positive rate (TPR), false positive rate (FPR) and Matthew's correlation coefficient (MCC) [22] to quantify the performance of methods, which are defined as:

$$
\begin{aligned}
T P R & =\frac{T P}{T P+F N} \\
F P R & =\frac{F P}{F P+T N} \\
M C C & =\frac{T P \times T N-F P \times F N}{\sqrt{(T P+F P)(T P+F N)(T N+F P)(T N+F N)}},
\end{aligned}
$$

where TP is the number of true positives, TN is the number of true negatives, FP is the number of false positives and FN is the number of false negatives. A TP decision assigns two related nodes to the same module. A TN decision assigns two unrelated nodes to different modules. An FP decision assigns two unrelated nodes to the same module. An FN decision assigns two related nodes to different modules. MCC returns a value in $[-1,1]$. A value of +1 represents a perfect prediction,
0 is no better than random prediction and -1 indicates total disagreement between prediction and observation.

\section{Performance}

We generate synthetic datasets with different value of $\alpha$. For our method, we use the parameters $\lambda_{s}=0.01, \lambda_{p}=0.05$ and $\theta=2$. The effects of parameters will be discussed later in more detail. All experiments are repeated 50 times on random generated datasets and the average results are reported for consistency. Figure 2 shows the examples of synthetic multiple networks with different type of conserved modules and the accuracy of each method in terms of TPR, FPR and MCC. ConMod outperforms the other methods in various value of $\alpha$ whenever the conserved modules are common to a given set of networks (Fig. 2a) or are the overlapping parts of specific modules across different networks (Fig. 2b). In particular, ConMod performs the best when the module structures are fuzzier $(\alpha=0.1)$.

Next, we evaluate the efficiency of ConMod. We conduct the experiments on a $2.10 \mathrm{GHz}$ desktop with $128 \mathrm{~GB}$ memory. Figure 3a shows the running time when varying the number of nodes and keeping the number of networks as 50 . Figure $3 \mathrm{~b}$ shows the running time when varying the number of networks and keeping each network size as 10,000. We do not compare with NetsTensor and omit the results of SC-ML, PairwiseCSRC and CentroidCSRC when the number of nodes is larger than 10,000 because of their high memory and running time cost. As can be seen from Fig. 3, the running time of ConMod is very low and is almost not affected by the number of networks, especially in large scale multiple networks. Additional figures regarding the other number of networks and nodes are put in the additional file (Additional file 1: Figure S1 and S2). 


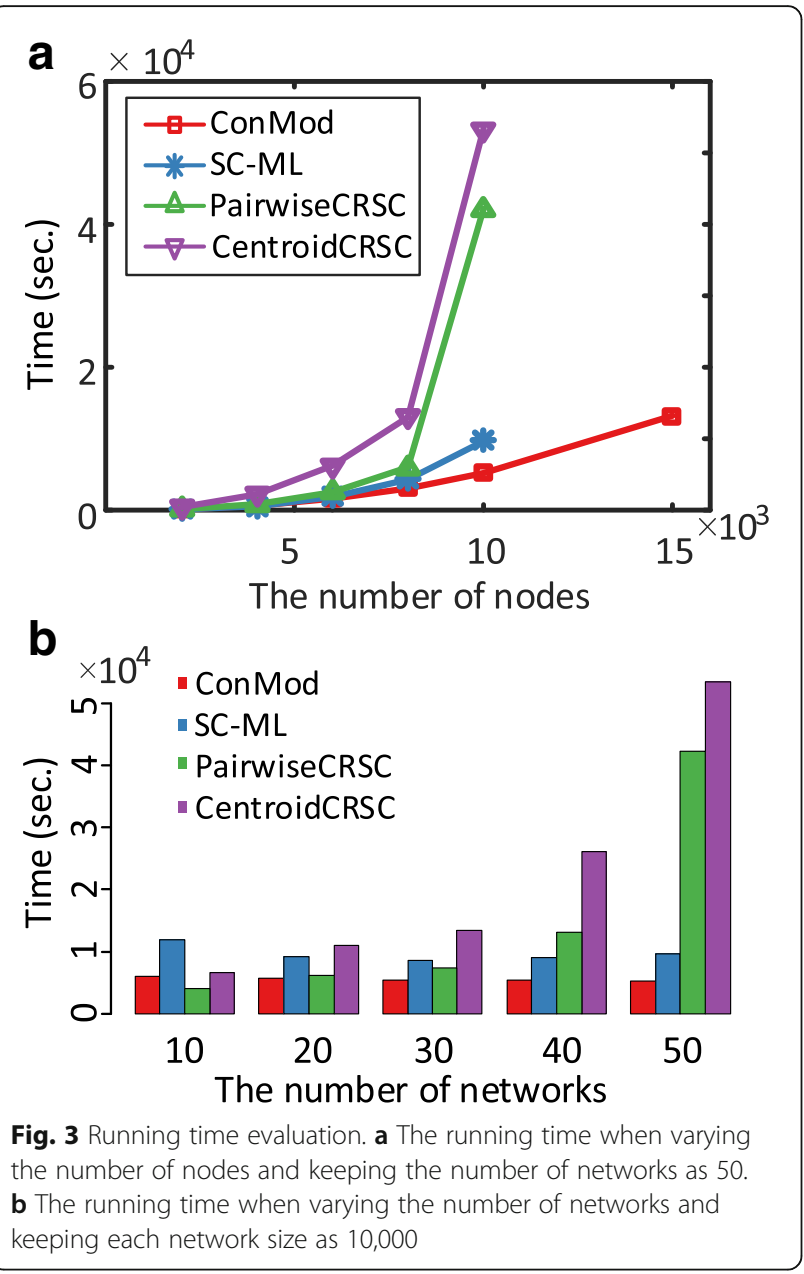

\section{Conserved functional modules in cancer type-specific gene co-expression networks}

In this section, we apply ConMod to multiple large-scale gene co-expression networks of 33 cancers. We aim at finding common signatures and biological functions in different cancers by identifying conserved functional modules. Such conserved gene co-expression modules can help reveal the gene expression regulatory basis for common traits in cancer [23].

We download the mRNA-sequencing data of all available 33 cancer types from The Cancer Genome Atlas (TCGA) database (https://portal.gdc.cancer.gov/). For each cancer type, we only select samples labeled as tumor. The Fragments Per Kilobase Million (FPKM) of each gene is transformed by $\log _{2}(\mathrm{FPKM}+1)$. For each cancer type, coding genes with FPKM $>1$ in more than $50 \%$ of all samples are selected. Then the intersection of expressed genes in all cancer types are used for constructing cancer type-specific gene co-expression networks based on Pearson's correlation coefficient. Meaningful relations are selected based on first-order partial correlation and information theory by PCIT R package [24]. Finally, we obtain a set of 33 cancer type-specific gene co-expression networks with 7,526 genes for each network.

We compare the performance of ConMod with NetsTensor [11], SC-ML [16], pairwiseCRSC [15] and centroidCRSC [15] by assessing the biological relevance of identified conserved functional modules. Here, we perform systematic enrichment analysis for genes of each module using Gene Ontology (GO) biological process $[25,26]$. We use precision, recall and f-score as the evaluation measures in this case. Precision is defined as the fraction of predicted modules that significantly overlap with reference gene sets. Recall is defined as the fraction of reference gene sets that significantly overlaps with predicted modules. F-score is defined as the harmonic mean of precision and recall. We calculate statistical significance $p$-value using Fisher's exact test and raw $p$-values were corrected using the method of Benjamin-Hochberg [21].

Figure 4 shows the performance of ConMod and other methods in terms of precision, recall and f-score w.r.t. different number of candidate modules $k$. We clearly see that ConMod is more stable than other methods and performs the best in most cases. Besides, we can see that the f-score is high enough when $k=150$ and has no significant increase after $k=150$, which can provide a reference for the selection of $k$. Note that NetsTensor does not need to specify the number of modules in advance, however it does not perform well because of the low node coverage and high overlap between discovered modules.

Next, after parameter optimizations, we set $k=150$ and $\theta=3.5$ and obtain 150 conserved functional modules covering 7,182 genes. The average module size is 113.2. We evaluated the resulting gene modules using multiple gold-standard gene set annotations from MsigDB [27] of GSEA [28], including the biological process category of Gene Ontology (GO) [25, 26], Canonical pathways (CP), Biocarta [29], KEGG [30] and REACTOME [31]. ConMod achieves higher f-scores than other four methods using all reference sets (Fig. 5a). We find that $86(57 \%)$ and $60(40 \%)$ of conserved modules are significantly enriched in at least one $\mathrm{GO}$ biological process and KEGG pathway ( $\mathrm{BH}$-adjusted $p$-value $<0.05)$. We present the top five significant GO biological processes and KEGG pathways in Fig. 5c and d respectively. We observe that these biological functions are related to ribosome protein, energy metabolism, cell cycle and immune response. Most of these functions are necessary to maintain a cell's life. These modules, acting as housekeeping roles, universally expressed in different tissues. However, cancers require a great deal of DNA replication and protein synthesis. Thus, most of the conserved modules and their functions are also closely associated with cancer. In particular, two significant GO biological 

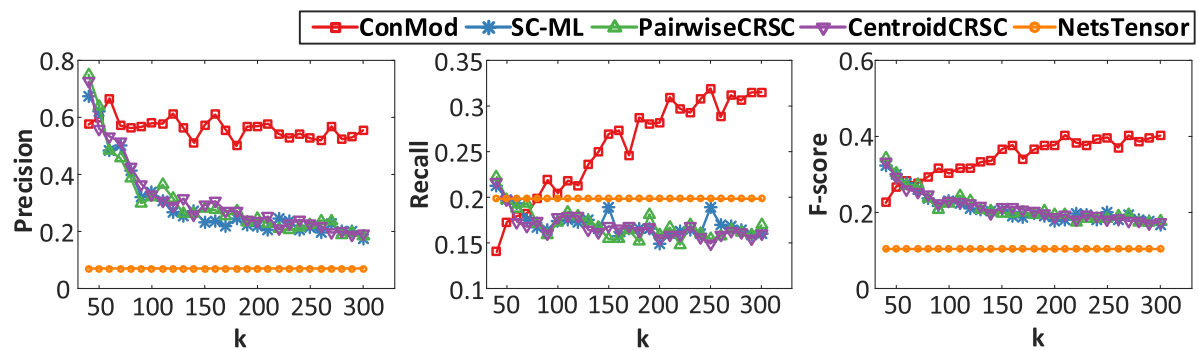

Fig. 4 Precision, recall and f-score with different $k$ in 33 cancer type-specific gene co-expression networks. Biological relevance of conserved modules is evaluated by GO biological process

processes, antigen processing and presentation and interferon-gamma-mediated signaling pathway, are both essential for immune response, which is often observed to be inhibited in the tumor microenvironment [7,32]. In addition, we test the relationship between the functional modules and cancer driver genes [33, 34]. By following a previous work [35], we utilized 2,372 genes from the Network of Cancer Genes (NCG) [36] as benchmarking cancer genes, including 711 known cancer genes from the Cancer Gene Census (CGC) [37]. We use Fisher's exact test to validate whether the modules are significantly associated with benchmark cancer
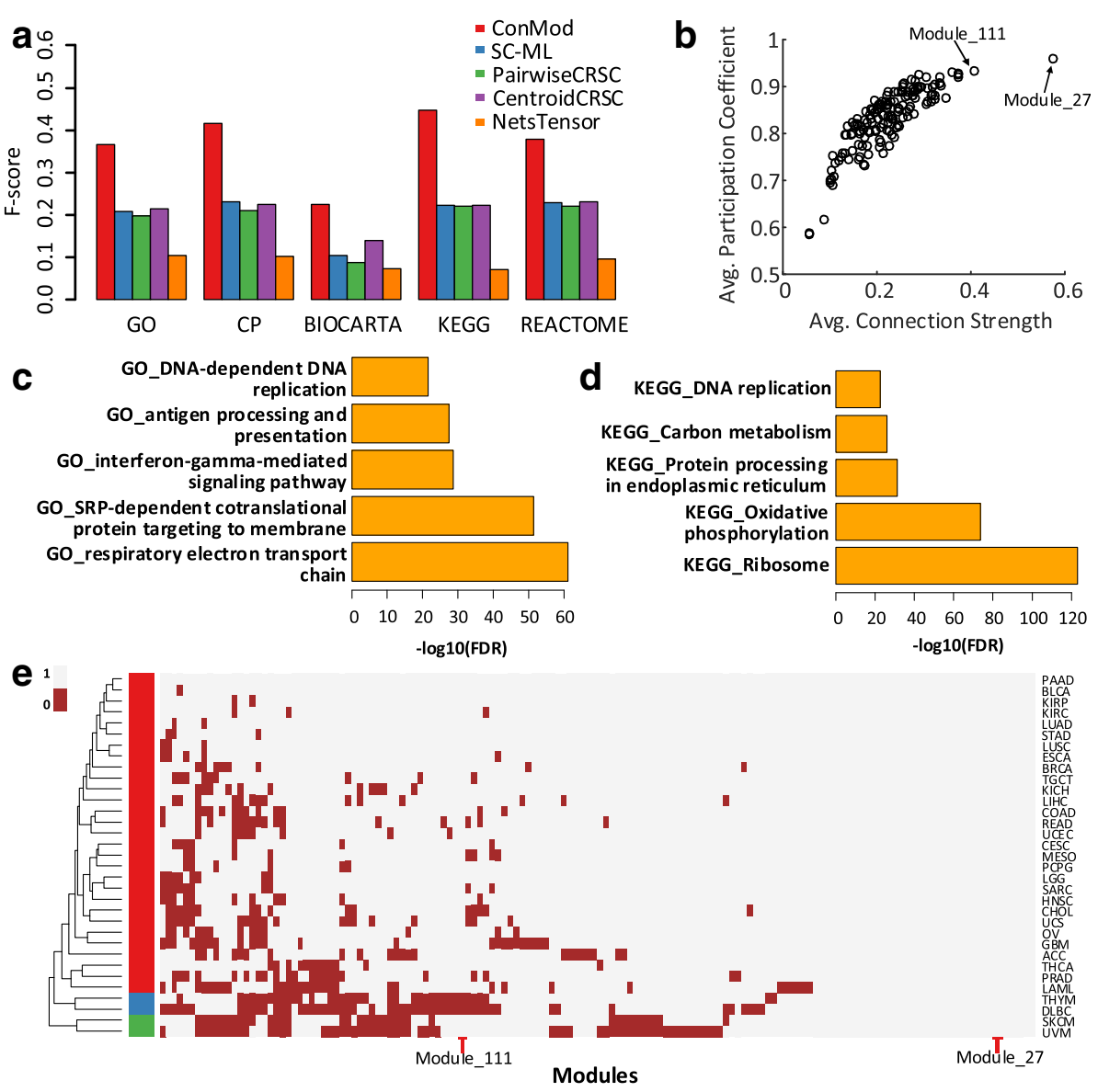

Fig. 5 Illustration of results on a set of 33 cancer type-specific gene co-expression networks. a F-score of five methods. Gene modules found by each method are evaluated by multiple gold-standard gene set annotations. $\mathbf{b}$ The scatter plot for the average value of connection strength and participation coefficient of each module. Each point represents an identified module. c Top five significant biological process enriched by the identified modules. $\mathbf{d}$ Top five significant KEGG pathways enriched by the identified modules. e Heat map of distribution of modules in multiple cancers. Each row represents a conserved module and each column corresponds to a cancer type. If a module significantly distributes in a cancer's network (BH-adjusted $p$-value $<0.01$, permutation test), it will be labeled as 1 . Otherwise, a module will be labeled as 0 
genes (BH-adjusted $p$-value $<0.05)$ and find that our method can get more modules with significantly enriched cancer driver genes than other methods (Additional file 1: Figure S3). This result indicates that the conserved functional modules identified by our method are able to reveal the characteristics of cancer.

We compute the average value of connection strength and participation coefficient respectively for each conserved module, and we observe that the two features are highly correlated (Pearson correlation coefficient $r=0.83$ ) (Fig. 5b). It is easily understood that a dense module conserved in more networks tend to has larger connection strength. After module validation, we can know how the conserved modules distribute in multiple networks (Fig. 5e). We consider that a module exists in a network if its Benjamin-Hochberg adjusted $p$-value $<0.01$ using a permutation test. Modules that do not exist in more than half of all networks are removed. From Fig. $5 \mathrm{e}$ we observe that about $25 \%$ of identified modules are common in all cancers and almost all modules are conserved in more than half of these cancers. Furthermore, similar cancers can be naturally clustered together only based on the distribution of identified modules (see the hierarchical clustering for cancers in Fig. 5e), such as SKCM (Skin Cutaneous Melanoma) and UVM (Uveal Melanoma); and THYM (Thymoma) and DLBC (Lymphoid Neoplasm Diffuse Large B-cell Lymphoma). Actually, SKCM and UVM are two types of melanoma, THYM and DLBC are both originated in the lymphatic system that participates in immune response.

Here, we take module 27 and module 111 as examples. Module 27, which has the largest connection strength and participation coefficient (Fig. 5b), significant exists in all cancers ( $\mathrm{BH}$-adjusted $p$-value $<0.01$, permutation test). This module contains 112 genes, among which 77 genes encode ribosomal protein (RP). RPs, which participate in ribosome composition, is widely distributed among various tissues. Ribosomes have the functions of DNA repair, cell development regulation and cell differentiation. In addition to their essential housekeeping roles in ribosome biogenesis and protein production in all cells, RPs were reported to change in the rate of ribosome biogenesis that regulate tumorigenesis [38-40]. In order to investigate the alterations gene expression patterns of this RP related gene module in different cancers, we compute the $\log 2$ fold-change for significantly differentially expressed genes in module 27 (Fig. 6b). 17 cancers with at least five normal samples are selected for this experiment. For each cancer, we use DESeq2 [41] to detect differentially expressed genes relative to normal samples. As shown in Fig. 6b, most genes of module 27 are significantly up-regulated in more than half cancers, especially in COAD (Colon Adenocarcinoma), LIHC (Liver Hepatocellular Carcinoma), PRAD (Portal
Prostate adenocarcinoma) and three kinds of kidney cancers (KIRC (Kidney renal clear cell carcinoma), KIRP (Kidney renal papillary cell carcinoma) and $\mathrm{KICH}$ (Kidney Chromophobe)). Even though cancer cells require continuous ribosome biogenesis and protein translation to maintain their high proliferation rate [39], it is reported that many RP genes have been found overexpressed in cancer and their mutations have been detected in the genome of cancer cells [40, 42], for example, in prostate cancer $[43,44]$ and in colorectal cancer $[45,46]$. Hence, targeting ribosome biogenesis of tumor cells could be an effective strategy [40].

Module 111 consist of 137 genes. Genes in this module mainly involve in antigen processing and neutrophil, leukocyte or $\mathrm{T}$ cell related processes, which are all closely related with cancers due to their important roles in immune system (Fig. 6c). This module, however, does not exist in THYM and DLBC (Fig. 5e). Actually, the module mainly splits into two dense sub-modules in THYM and DLBC respectively, but maintains a complete module in the rest cancers, e.g. in LUAD (Lung Adenocarcinoma) (Fig. 6d). In particular, module 111 in DLBC consist of a large sub-module and a small sub-module. The small part comprise 10 genes (CD74, HLA-DQB1, HLA-DRB1, HLA-DQA1, HLA-DRB5, HLA-DMA, HLA-DRA, HLA-DPB1, HLA-DPA1, HLA-DMB), all of which are MHC (major histocompatibility complex) class II genes in HLA (human leucocyte antigen). The separation of the two sub-modules results from the weak correlation in expression between the MHC class II genes in the small sub-module and other immune-related genes in the large sub-module, suggesting a disruption of the co-operation of these genes to exert immunity responses. Actually, DLBC is a cancer of $B$ cells. Cancerous B cells can not normally produce MHC class II molecules, which are exported to B cell's surface and interact with their intended $\mathrm{T}$ cells to initiate immune response [47].

\section{Conserved function modules in human brain tissue- specific interaction networks}

The human brain is a complex system organized by structural and functional relationships between its functional regions, such as the thalamus, brainstem and other brain tissues. Recently, multiple brain networks and their applications in neuroscience have successfully uncovered brain-associated features [48, 49]. We now aim to identify conserved protein modules across human tissue-specific networks, which may reveal important function units for brain activity.

We run ConMod on a set of 15 human brain tissue-specific protein interaction networks [50] to find conserved protein modules. There are 2,721 proteins in total. It should be noted that, different from 33 cancer 

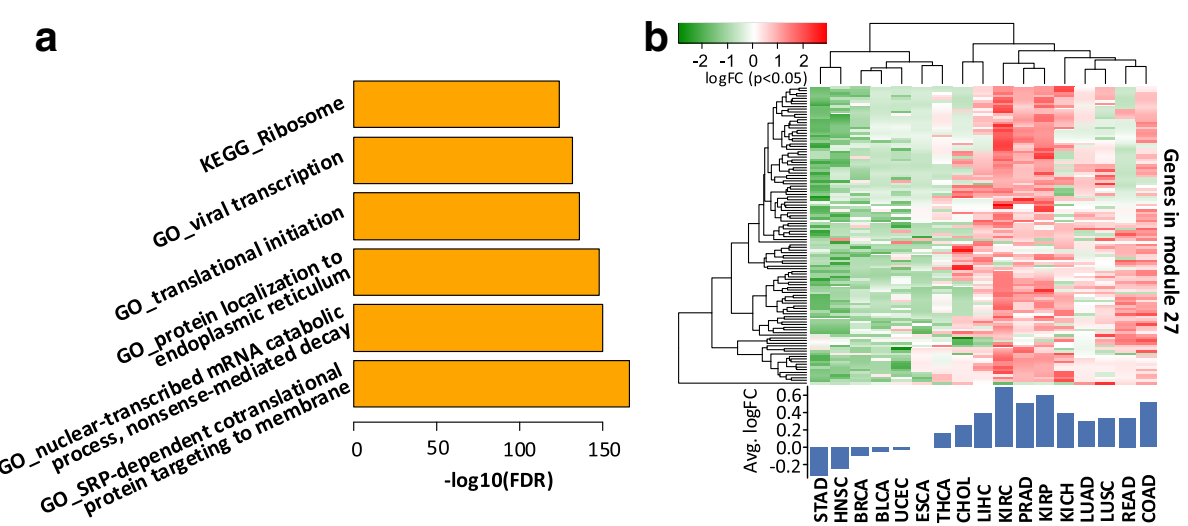

C
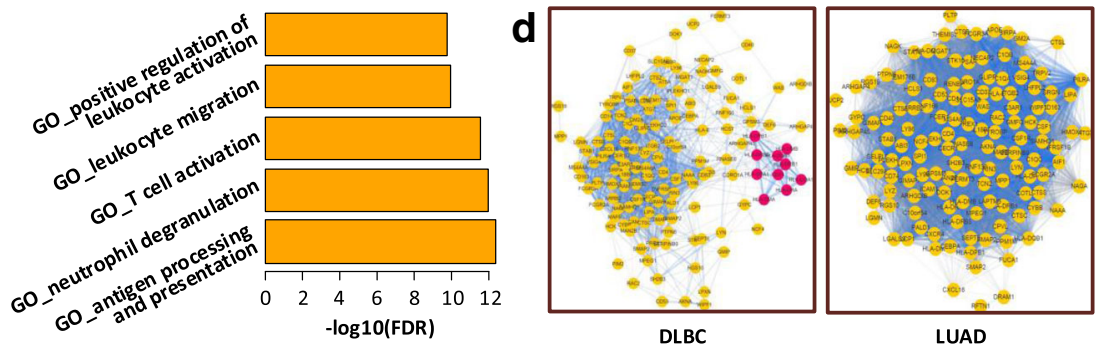

Fig. 6 Illustration of module 27 and module 111. a Top significant biological terms enriched by the genes of module 27. The top five GO terms in biological process and the only one enriched KEGG pathway are displayed. $\mathbf{b}$ The heat map illustrates the log2 fold-change of gene expression in module 27 for each cancer relative to normal samples. The bar plot under the heat map shows the average log2 fold-change of all genes in module 27. Cancers with at least five normal samples are selected. c Top significant GO terms in biological process enriched by the genes of module 111. $\mathbf{d}$ The presentations of module 111 in DLBC and LUAD. The module mainly split into two dense sub-modules in DLBC, a small part consists of MHC class II genes and a large part consists other immune-related genes

type-specific networks in the above section, all networks of this dataset are unweighted and they have different number of nodes.

We compare ConMod with SC-ML and NetsTensor on this data because other methods are not suitable for the dataset in which the set of data objects is different in each network. Figure 7 shows the performance of ConMod and other methods in terms of precision, recall and f-score w.r.t. different number of candidate modules $k$. As is shown, ConMod outperforms SC-ML and NetsTensor in precision for all settings of $k$ while maintaining comparable recall values. As an average, ConMod has a better performance in $\mathrm{f}$-score.
After parameter optimizations, we set $k=120$ and $\theta=4$ and obtained 114 conserved functional modules covering 1,414 genes. The average module size is 23.2 . We evaluated these modules using multiple gold-standard gene set annotations as the same procedure mentioned in the above section. As shown in Fig. 8a, ConMod achieves higher f-score when evaluated using all reference sets. The identified conserved modules mainly relate to nervous system development, mRNA processing, etc. (Additional file 1: Figure S4). Here, we take module 7 as an example (Fig. 8c). Module 7, which has the largest connection strength and participation coefficient in this dataset (Fig. 8b), consists of seven proteins with a
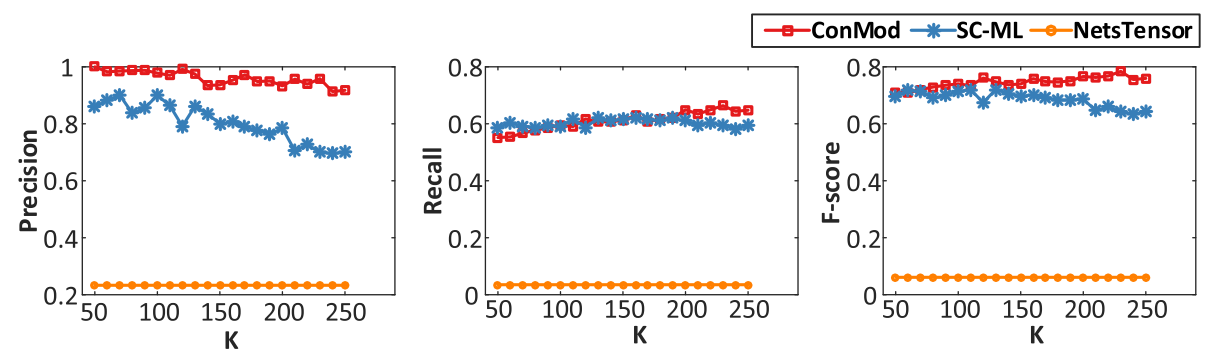

Fig. 7 Precision, recall and f-score with different $k$ in 15 brain tissue-specific protein interaction networks. Modules are evaluated by GO biological process 

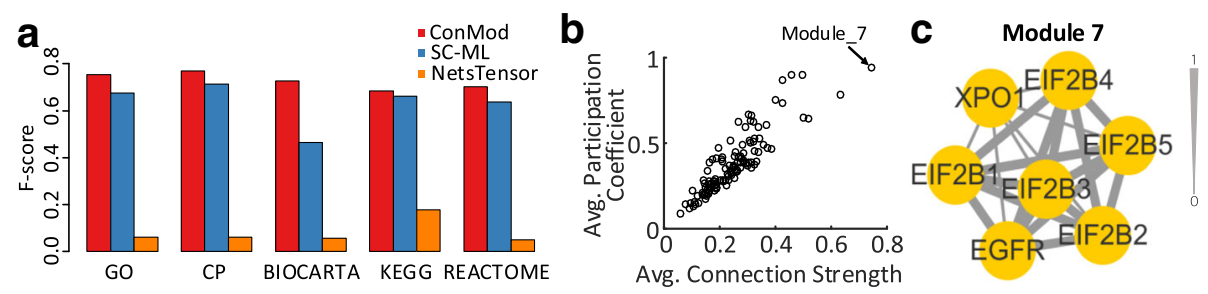

Fig. 8 Illustration of results on 15 brain tissue-specific interaction networks. a F-score of three methods. Gene modules found by each method are evaluated by multiple gold-standard gene set annotations. $\mathbf{b}$ The scatter plot for the average value of connection strength and participation coefficient of each module. c A case of identified conserved module that are significant related with glial cell development (FDR $=3.94 \mathrm{E}-11)$

significant number (6) of proteins (EIF2B1, EIF2B2, EIF2B3, EIF2B4, EIF2B5 and EGFR) for glial cell development $(\mathrm{BH}$-adjusted $p$-value $=3.94 \mathrm{E}-11)$. Leegwater et al. [51] reported that the gene mutations of the five subunit proteins (EIF2B1, EIF2B2, EIF2B3, EIF2B4 and EIF2B5) of EIF2B complex can lead to white matter abnormalities, a serious hereditary neurodegenerative disease. Another important gene is EGFR, which is widely distributed in glial cells of mammalian brain. EGFR activation is essential for the proliferation of multipotent neural precursors, as well as the survival, migration, and differentiation of the immature daughter cells [52].

\section{Parameter discussion}

There are four parameters in our ConMod method: the regularization parameters $\lambda_{s}$ and $\lambda_{p}$ in multi-view NMF, the number of modules $k$ and the threshold $\theta$ for nodes selection. We first discuss the influence of parameters $\lambda_{s}$ and $\lambda_{p}$. Following a similar approach as proposed in Ref. [14], we set $\lambda_{v}$ to be the same for convenience, that is $\lambda_{v}$ $=\lambda_{s}=\lambda_{p}$, and varying it from $10^{-3}$ to 1 on two synthetic datasets with $\alpha=0.1$ and $\alpha=0.3$. The optimal values appear when $\lambda_{v}$ is around 0.01 and the accuracy is relatively stable when $\lambda_{v}<0.1$ (Additional file 1: Figure S5). We aim at finding conserved modules, thus we let the participation coefficient has a larger effect by denoting $\lambda_{s}=0.01$ and $\lambda_{p}=0.05$ for all the experiments.

The selection of the parameter $k$ has a significant effect on the results. While the choice of $k$ is often data-dependent and is a long-standing open problem. The lower $k$ of the reduced space is a key parameter for this study. For the synthetic datasets, we set $k$ as the real number of modules. However, for real biological datasets that the real number of modules is unknown, we select a proper $k$ by assessing the enrichment rate of gene modules with respect to $\mathrm{GO}$ biological process. A low $k$ with a relative high $\mathrm{f}$-score is selected for each datasets. We have shown in the experiments that we choose $k=150$ for multiple co-expression networks of cancers (Fig. 4; Additional file 1: Figure S6) and $k=120$ for multiple brain-specific protein interaction networks (Fig. 7; Additional file 1: Figure S7).

The parameter $\theta$ determines the size of a module. A larger $\theta$ means a small size of module, but a more significant signal in the consensus factors. $\theta$ is generally larger than 2 , because the corresponding $p$-value is smaller than 0.05 . In our experiments we choose $\theta=2$ for all the synthetic datasets, $\theta=3.5$ for multiple co-expression networks of cancers (Additional file 1: Figure S6) and $\theta=4$ for multiple brain-specific protein interaction networks (Additional file 1: Figure S7). The reason for selection these values of parameter $\theta$ is that we try to keep small size of modules and a high coverage of total number of nodes while ensure a high accuracy.

\section{Conclusion}

In this study, we present ConMod, a method for identifying conserved functional modules in multiple biological networks. Experiments on two types of simulated data show that ConMod has competitive performance in accuracy and efficiency when compared with four state-of-the-art methods. Effectiveness of ConMod on real biological networks is further demonstrated using cancer type-specific gene co-expression networks and brain tissue-specific protein interaction networks. The major advantage of our approach is that the proposed two features, connection strength and participation coefficient, give a new insight into characterizing the structure of multiple networks, which compress multiple networks without a lot of information loss. Furthermore, ConMod is very flexible for identifying conserved modules in multiple networks, because it can be applied to a set of any number of unweighted and weighted networks, and it can also be easily extended to other types of networks outside biology.

Besides the importance of conserved modules in biological networks, specific modules also have great significance for better understanding biological mechanism and even for precision medicine. Although the introduced two features are helpful for mining conserved modules, they might not fully characterize the module 
structures in multiple networks. Thus, it is crucial to design an algorithm to detect conserved modules and condition-specific modules simultaneously. We hope that future work on integrating more types of biological networks will provide greater insight into pathway structures and highlight network-level dynamics underlying biological responses.

\section{Additional file}

Additional file 1: Supplement containing information on the multi-view symmetric NMF method, the construction of synthetic networks and figures about additional results. (PDF $336 \mathrm{~kb}$ )

\section{Abbreviations}

BH: Benjamin-Hochberg; COAD: Colon Adenocarcinoma; CP: Canonical pathways; DLBC: Lymphoid Neoplasm Diffuse Large B-cell Lymphoma; FPKM: Fragments Per Kilobase Million; FPR: False positive rate; GO: Gene Ontology; HLA: Human leucocyte antigen; KICH: Kidney Chromophobe; KIRC: Kidney renal clear cell carcinoma; KIRP: Kidney Renal Papillary Cell Carcinoma; LIHC: Liver Hepatocellular Carcinoma; LUAD: Lung Adenocarcinoma; MCC: Matthew's correlation coefficient; MHC: Major histocompatibility complex; NMF: Non-negative matrix factorization; PRAD: Portal Prostate Adenocarcinoma; RP: Ribosomal protein; SKCM: Skin Cutaneous Melanoma; TCGA: The Cancer Genome Atlas; THYM: Thymoma; TPR: True positive rate; UVM: Uveal Melanoma

\section{Acknowledgements}

We would like to thank members of the Lin Gao Lab for their helpful comments and suggestions. We also thank all the guest editors and anonymous reviewers for their constructive comments on the manuscript.

\section{Funding}

This work was supported by the National Natural Science Foundation of China (Grant No.61532014, No.61432010 and No.61702397). The funding organizations did not play any role in the design of the study, data collection and analysis, or preparation of the manuscript.

\section{Availability of data and materials}

All cancer related data analyzed in this research was obtained from The Cancer Genome Atlas, which is now available in The Genomic Data Commons Data Portal (https://portal.gdc.cancer.gov/). The human brain tissue-specific protein interaction networks were reported in [50] and is available in http://snap. stanford.edu/ohmnet/. ConMod is implemented in MATLAB R2015a as a userfriendly package and is available in https://github.comNPPZithub/ConMod.

\section{Authors' contributions}

PZW designed the method and carried out all programming work. LG initiated, supervised the project and participated in the data analysis. YXH and FL provided helpful information from the perspective of biology. All authors discussed the results and wrote the manuscript. All authors read and approved the final manuscript.

\section{Ethics approval and consent to participate}

Not applicable

\section{Consent for publication}

Not applicable

\section{Competing interests}

The authors declare that they have no competing interests.

\section{Publisher's Note}

Springer Nature remains neutral with regard to jurisdictional claims in published maps and institutional affiliations.
Received: 8 May 2018 Accepted: 15 October 2018

Published online: 29 October 2018

\section{References}

1. Bersanelli M, Mosca E, Remondini D, Giampieri E, Sala C, Castellani G, Milanesi L. Methods for the integration of multi-omics data: mathematical aspects. BMC Bioinf. 2016;17(2):S15.

2. Boccaletti S, Bianconi G, Criado R, Del Genio Cl, Gómez-Gardenes J, Romance M, Sendina-Nadal I, Wang Z, Zanin M. The structure and dynamics of multilayer networks. Phys Rep. 2014;544(1):1-122.

3. Kivelä M, Arenas A, Barthelemy M, Gleeson JP, Moreno Y, Porter MA. Multilayer networks. J Complex Networks. 2014;2(3):203-71.

4. Yugi K, Kubota H, Hatano A, Kuroda S. Trans-omics: how to reconstruct biochemical networks across multiple 'omic'layers. Trends Biotechnol. 2016; 34(4):276-90.

5. Gosak M, Markovič R, Dolenšek J, Slak Rupnik M, Marhl M, Stožer A, Perc M. Network science of biological systems at different scales: A review. Physics of Life Reviews. 2018;24:118-35.

6. Weinstein JN, Collisson EA, Mills GB, Shaw KRM, Ozenberger BA, Ellrott K, Shmulevich I, Sander C, Stuart JM, Network CGAR. The cancer genome atlas pan-cancer analysis project. Nat Genet. 2013;45(10):1113.

7. Hanahan D, Weinberg RA. Hallmarks of cancer: the next generation. cell. 2011;144(5):646-74.

8. Gerstein MB, Rozowsky J, Yan K-K, Wang D, Cheng C, Brown JB, Davis CA, Hillier L, Sisu C, Li JJ. Comparative analysis of the transcriptome across distant species. Nature. 2014;512(7515):445.

9. Yan K-K, Wang D, Rozowsky J, Zheng H, Cheng C, Gerstein M. OrthoClust: an orthology-based network framework for clustering data across multiple species. Genome Biol. 2014;15(8):R100.

10. Hu H, Yan X, Huang Y, Han J, Zhou XJ. Mining coherent dense subgraphs across massive biological networks for functional discovery. Bioinformatics. 2005;21(suppl_1):i213-21.

11. Li WY, Liu CC, Zhang T, Li HF, Waterman MS, Zhou XHJ. Integrative analysis of many weighted co-expression networks using tensor computation. PLoS Comput Biol. 2011;7(6):Cp8-U20.

12. Xiao X, Moreno-Moral A, Rotival M, Bottolo L, Petretto E: Multi-tissue analysis of co-expression networks by higher-order generalized singular value decomposition identifies functionally coherent transcriptional modules. Plos Genet. 2014;10(1):e1004006.

13. Huang H-C, Chuang Y-Y, Chen C-S: Affinity aggregation for spectral clustering. In: 2012 IEEE Conference on Computer Vision and Pattern Recognition (CVPR): 16-21 June 2012; Providence. Rl: IEEE; 2012. p. 773-780.

14. Liu J, Wang C, Gao J, Han J: Multi-view clustering via joint nonnegative matrix factorization. In: Proceedings of the 2013 SIAM International Conference on Data Mining: 2-4 May 2013. Austin: SIAM; 2013. p. 252-260.

15. Tsivtsivadze E, Borgdorff $H$, van de Wijgert J, Schuren F, Verhelst R, Heskes T. Neighborhood co-regularized multi-view spectral clustering of microbiome data. Lect Notes Artif Int. 2013;8193:80-90.

16. Dong XW, Frossard P, Vandergheynst P, Nefedov N. Clustering on multilayer graphs via subspace analysis on grassmann manifolds. leee T Signal Proces. 2014;62(4):905-18.

17. Guimerà R, Nunes Amaral LA: Cartography of complex networks: modules and universal roles. Journal of Statistical Mechanics: Theory and Experiment. 2005;2005(02):P02001.

18. Guimerà R, Nunes Amaral LA: Functional cartography of complex metabolic networks. Nature. 2005; 433:895-900.

19. $\mathrm{Ni} \mathrm{J}$, Tong H, Fan W, Zhang X. Flexible and robust multi-network clustering. In: Proceedings of the 21th ACM SIGKDD International Conference on Knowledge Discovery and Data Mining: August 10-13 2015. Sydney: ACM; 2015. p. 835-844.

20. Zong L, Zhang $X$, Zhao L, Yu H, Zhao Q. Multi-view clustering via multimanifold regularized non-negative matrix factorization. Neural Netw. 2017; 88:74-89.

21. Benjamini Y, Hochberg Y. Controlling the false discovery rate: A practical and powerful approach to multiple testing. J. R. Stat Soc Ser B Methodol. 1995:57(1):289-300.

22. Matthews BW. Comparison of the predicted and observed secondary structure of T4 phage lysozyme. Biochim Biophys Acta (BBA) Protein Struct. 1975;405(2):442-51.

23. Zhang J, Lu K, Xiang Y, Islam M, Kotian S, Kais Z, Lee C, Arora M, H-w L, Parvin JD, et al. Weighted frequent gene co-expression network mining to 
identify genes involved in genome stability. PLoS Comput Biol. 2012;8(8): e1002656.

24. Watson-Haigh NS, Kadarmideen HN, Reverter A. PCIT: an R package for weighted gene co-expression networks based on partial correlation and information theory approaches. Bioinformatics. 2009;26(3):411-3.

25. Ashburner M, Ball CA, Blake JA, Botstein D, Butler H, Cherry JM, Davis AP, Dolinski K, Dwight SS, Eppig JT. Gene ontology: tool for the unification of biology. Nat Genet. 2000;25(1):25-9.

26. Consortium GO. Expansion of the gene ontology knowledgebase and resources. Nucleic Acids Res. 2016:45(D1):D331-8.

27. Liberzon A, Birger C, Thorvaldsdóttir H, Ghandi M, Mesirov Jill P, Tamayo P. The molecular signatures database hallmark gene set collection. Cell Syst. 2015;1(6):417-25.

28. Subramanian A, Tamayo P, Mootha VK, Mukherjee S, Ebert BL, Gillette MA, Paulovich A, Pomeroy SL, Golub TR, Lander ES, et al. Gene set enrichment analysis: a knowledge-based approach for interpreting genome-wide expression profiles. Proc Natl Acad Sci. 2005;102(43):15545.

29. Nishimura D. BioCarta. Biotech Software Internet Rep. 2001;2(3):117-20.

30. Kanehisa M, Goto S, Sato Y, Furumichi M, Tanabe M. KEGG for integration and interpretation of large-scale molecular data sets. Nucleic Acids Res. 2012;40(D1):D109-14.

31. Fabregat A, Jupe S, Matthews L, Sidiropoulos K, Gillespie M, Garapati P, Haw $R$, Jassal B, Korninger F, May B, et al. The Reactome pathway knowledgebase. Nucleic Acids Res. 2018;46(D1):D649-55.

32. Whiteside TL. Immune suppression in cancer: Effects on immune cells, mechanisms and future therapeutic intervention. Semin Cancer Biol. 2006; 16(1):3-15.

33. Xi J, Wang M, Li A. Discovering mutated driver genes through a robust and sparse co-regularized matrix factorization framework with prior information from mRNA expression patterns and interaction network. BMC Bioinf. 2018; 19(1):214.

34. Xi J, Li A, Wang M. A novel unsupervised learning model for detecting driver genes from pan-cancer data through matrix tri-factorization framework with pairwise similarities constraints. Neurocomputing. 2018;296:64-73.

35. Xi J, Wang M, Li A. Discovering potential driver genes through an integrated model of somatic mutation profiles and gene functional information. Mol BioSyst. 2017;13(10):2135-44.

36. Kuppili Venkata S, Repana D, Nulsen J, Dressler L, Bortolomeazzi M, Tourna A, Yakovleva A, Palmieri T, Ciccarelli FD. The network of Cancer genes (NCG): a comprehensive catalogue of known and candidate cancer genes from cancer sequencing screens. In: bioRxiv; 2018.

37. Futreal PA, Coin L, Marshall M, Down T, Hubbard T, Wooster R, Rahman N, Stratton MR. A census of human cancer genes. Nat Rev Cancer. 2004:4:177.

38. de las Heras-Rubio A, Perucho L, Paciucci R, Vilardell J, LLeonart ME. Ribosomal proteins as novel players in tumorigenesis. Cancer Metastasis Rev. 2014:33(1):115-41.

39. Takada $\mathrm{H}$, Kurisaki A. Emerging roles of nucleolar and ribosomal proteins in cancer, development, and aging. Cell Mol Life Sci. 2015;72(21):4015-25.

40. Zhou X, Liao W-J, Liao J-M, Liao P, Lu H. Ribosomal proteins: functions beyond the ribosome. J Mol Cell Biol. 2015;7(2):92-104.

41. Love Ml, Huber W, Anders S. Moderated estimation of fold change and dispersion for RNA-seq data with DESeq2. Genome Biol. 2014;15(12):550

42. Goudarzi KM, Lindström MS. Role of ribosomal protein mutations in tumor development. Int J Oncol. 2016;48(4):1313-24.

43. Bee A, Ke Y, Forootan S, Lin K, Beesley C, Forrest SE, Foster CS. Ribosomal protein 119 is a prognostic marker for human prostate cancer. Clin Cancer Res. 2006;12(7):2061-5.

44. Vaarala MH, Porvari KS, Kylloenen AP, Mustonen MV, Lukkarinen O, Vihko PT. Several genes encoding ribosomal proteins are over-expressed in prostatecancer cell lines: confirmation of L7a and L37 over-expression in prostatecancer tissue samples. Int J Cancer. 1998;78:27-32.

45. Pogue-Geile K, Geiser JR, Shu M, Miller C, Wool IG, Meisler Al, Pipas JM. Ribosomal protein genes are overexpressed in colorectal cancer: isolation of a cDNA clone encoding the human S3 ribosomal protein. Mol Cell Biol. 1991:11(8):3842-9.

46. Mao-De L, Jing X. Ribosomal proteins and colorectal cancer. Curr Genomics. 2007:8(1):43-9.

47. Yuseff M-I, Pierobon P, Reversat A, Lennon-Duménil A-M. How B cells capture, process and present antigens: a crucial role for cell polarity. Nat Rev Immunol. 2013:13:475.
48. De Domenico M. Multilayer modeling and analysis of human brain networks. GigaScience. 2017;6(5):1-8.

49. Vaiana M, Muldoon SF. Multilayer brain networks. Journal of Nonlinear Science. 2018:2018:1-23.

50. Zitnik M, Leskovec J. Predicting multicellular function through multi-layer tissue networks. Bioinformatics. 2017;33(14):i190-8.

51. Leegwater PA, Vermeulen G, Könst AA, Naidu S, Mulders J, Visser A, Kersbergen $P$, Mobach D, Fonds D, van Berkel CG. Subunits of the translation initiation factor elF2B are mutant in leukoencephalopathy with vanishing white matter. Nat Genet. 2001;29(4):383.

52. Estrada C, Villalobo A. Epidermal growth factor receptor in the adult brain In: Janigro D, editor. The cell cycle in the central nervous system. Totowa, NJ: Humana Press; 2006. p. 265-77.
Ready to submit your research? Choose BMC and benefit from:

- fast, convenient online submission

- thorough peer review by experienced researchers in your field

- rapid publication on acceptance

- support for research data, including large and complex data types

- gold Open Access which fosters wider collaboration and increased citations

- maximum visibility for your research: over $100 \mathrm{M}$ website views per year

At BMC, research is always in progress.

Learn more biomedcentral.com/submissions 